\title{
CENTRALLY SYMMETRIC ORTHOGONAL POLYNOMIALS AND SECOND ORDER PARTIAL DIFFERENTIAL EQUATIONS*
}

\author{
Y. J. KIM ${ }^{\dagger}$, K. H. KWON ${ }^{\ddagger}$, AND J. K. LEE ${ }^{\S}$
}

Abstract. We classify completely, up to a real change of variables, all differential equations

$$
L[u]:=A u_{x x}+2 B u_{x y}+C u_{y y}+D u_{x}+E u_{y}=\lambda_{n} u,
$$

which have centrally symmetric orthogonal polynomial solutions.

1. Introduction and Preliminaries. Consider a second order partial differential equations of the type

$$
L[u]:=A u_{x x}+2 B u_{x y}+C u_{y y}+D u_{x}+E u_{y}=\lambda_{n} u, \quad n=0,1,2, \cdots,
$$

where $A \sim E$ are polynomials in $x$ and $y$. Krall and Sheffer[5] classified equations (1.1), up to a complex linear change of variables, which have orthogonal polynomials as solutions.

However, complex linear change of variables does not preserve the positivedefiniteness of orthogonality and the type of the equation (1.1). In this respect, we classify completely, up to a real change of variables, the equations (1.1) which have centrally symmetric orthogonal polynomials as solutions together with explicit representations of orthogonal polynomial solutions.

For any integer $n \geq 0$, let $\mathcal{P}_{n}$ be the space of real polynomials in two variables of (total) degree $\leq n$ and $\mathcal{P}=\bigcup_{n \geq 0} \mathcal{P}_{n}$. By a polynomial system(PS), we mean a sequence of polynomials $\left\{\phi_{m n}\right\}_{m, n=0}^{\infty}$ such that $\operatorname{deg}\left(\phi_{m n}\right)=m+n$ for $m$ and $n \geq 0$ and $\left\{\phi_{n-j, j}\right\}_{j=0}^{n}$ are linearly independent modulo $\mathcal{P}_{n-1}$ for $n \geq 0\left(\mathcal{P}_{-1}=\{0\}\right)$. A PS $\left\{P_{m n}\right\}_{m, n=0}^{\infty}$ is said to be monic if

$$
P_{m n}(x, y)=x^{m} y^{n} \text { modulo } \mathcal{P}_{m+n-1}, \quad m \text { and } n \geq 0 .
$$

A linear mapping $\sigma: \mathcal{P} \rightarrow \mathbb{R}$ is called a moment functional, whose action on a polynomial $\phi \in \mathcal{P}$ is denoted by $\langle\sigma, \phi\rangle$. For any moment functional $\sigma$, we define the partial derivatives $\sigma_{x}$ and $\sigma_{y}$ of $\sigma$ by

$$
\left\langle\sigma_{x}, \phi\right\rangle:=-\left\langle\sigma, \phi_{x}\right\rangle, \quad\left\langle\sigma_{y}, \phi\right\rangle:=-\left\langle\sigma, \phi_{y}\right\rangle \quad(\phi \in \mathcal{P}),
$$

and the multiplication $\psi \sigma$ for $\psi \in \mathcal{P}$ by $\langle\psi \sigma, \phi\rangle:=\langle\sigma, \psi \phi\rangle$.

DEFINITION 1.1. ([5]) A PS $\left\{\phi_{m n}\right\}_{m, n=0}^{\infty}$ is a weak orthogonal polynomial system $(W O P S)$ if there is a non-zero moment functional $\sigma$ such that $\left\langle\sigma, \phi_{m n} \phi_{k l}\right\rangle=0$, if $m+$ $n \neq k+l$.

If furthermore

$$
\left\langle\sigma, \phi_{m n} \phi_{k l}\right\rangle=K_{m n} \delta_{m k} \delta_{n l}
$$

where $K_{m n}$ are non-zero(resp., positive) constants, we call $\left\{\phi_{m n}\right\}_{m, n=0}^{\infty}$ an orthogonal polynomial system(OPS) (resp., a positive-definite OPS). In this case, we say that $\left\{\phi_{m n}\right\}_{m, n=0}^{\infty}$ is a WOPS or an OPS relative to $\sigma$.

${ }^{*}$ Received April 21, 1999; revised Nov. 15, 1999.

†Department of Mathematics, KAIST, Taejon 305-701, Korea (khkwon@jacobi.kaist.ac.kr).

$\ddagger$ Department of Mathematics, KAIST, Taejon 305-701, Korea.

$\S$ Department of Mathematics, Sunmoon university, Asan, Korea (jklee@omega.sunmoon.ac.kr). 
For any PS $\left\{\phi_{m n}\right\}_{m, n=0}^{\infty}$, there is a unique moment functional $\sigma$, called the canonical moment functional of $\left\{\phi_{m n}\right\}_{m, n=0}^{\infty}$, defined by the conditions

$$
\langle\sigma, 1\rangle=1 \text { and }\left\langle\sigma, \phi_{m n}\right\rangle=0, m+n \geq 1 \text {. }
$$

In the following, we write a PS $\left\{\phi_{m n}\right\}_{m, n=0}^{\infty}$ as $\left\{\Phi_{n}\right\}_{n=0}^{\infty}$ where $\Phi_{n}=\left[\phi_{n 0}, \phi_{n-1,1}\right.$, $\left.\cdots, \phi_{0 n}\right]^{T}$ and let $\mathbf{x}^{n}=\left[x^{n}, x^{n-1} y, \cdots, y^{n}\right]^{T}, n \geq 0$. When $\Phi_{n}=A_{n} \mathbf{x}^{n}$ modulo $\mathcal{P}_{n-1}$, we call the monic PS $\left\{\mathbb{P}_{n}\right\}_{n=0}^{\infty}$ the normalization of $\left\{\Phi_{n}\right\}_{n=0}^{\infty}$, where $\mathbb{P}_{n}:=A_{n}^{-1} \Phi_{n}$.

Definition 1.2. A moment functional $\sigma$ is quasi-definite (resp., positive-definite) if there is an OPS (resp., a positive-definite OPS) relative to $\sigma$.

Proposition 1.3. ([1,5]) For a moment functional $\sigma \neq 0, \sigma$ is quasidefinite(resp., positive-definite) if and only if $D_{n}$ is nonsingular (resp., positivedefinite), where

$$
D_{n}:=\left[\begin{array}{ccccccc}
\sigma_{00} & \sigma_{10} & \sigma_{01} & \cdots & \sigma_{n 0} & \cdots & \sigma_{0 n} \\
\sigma_{10} & \sigma_{20} & \sigma_{11} & \cdots & \sigma_{n+1,0} & \cdots & \sigma_{1 n} \\
\vdots & \vdots & \vdots & \ddots & \vdots & \ddots & \vdots \\
\sigma_{0 n} & \sigma_{1 n} & \sigma_{0, n+1} & \cdots & \sigma_{n n} & \cdots & \sigma_{0,2 n}
\end{array}\right], n \geq 0
$$

and $\sigma_{m, n}=\left\langle\sigma, x^{m} y^{n}\right\rangle, m$ and $n \geq 0$, are the moments of $\sigma$.

For any $\operatorname{PS}\left\{\Phi_{n}\right\}_{n=0}^{\infty}$, there are matrices

$$
\begin{array}{ll}
A_{n i}:(n+1) \times(n+2), & B_{n i}:(n+1) \times(n+1), \\
C_{n i}:(n+1) \times n, & D_{n i}^{k}:(n+1) \times(k+1)
\end{array}
$$

for $i=1,2$ and $k=0,1, \cdots, n-2$ such that

$$
\mathbf{x} \Phi_{n}:=\left[\begin{array}{l}
x \Phi_{n} \\
y \Phi_{n}
\end{array}\right]=A_{n} \Phi_{n+1}+B_{n} \Phi_{n}+C_{n} \Phi_{n-1}+\sum_{k=0}^{n-2} D_{n}^{k} \Phi_{k}
$$

where $A_{n}=\left[\begin{array}{l}A_{n 1} \\ A_{n 2}\end{array}\right], B_{n}=\left[\begin{array}{l}B_{n 1} \\ B_{n 2}\end{array}\right], C_{n}=\left[\begin{array}{c}C_{n 1} \\ C_{n 2}\end{array}\right], D_{n}^{k}=\left[\begin{array}{c}D_{n 1}^{k} \\ D_{n 2}^{k}\end{array}\right]$.

Note that since $\left\{\Phi_{n}\right\}_{n=0}^{\infty}$ is a PS, rank $A_{n}=n+2, n \geq 0$.

Proposition 1.4. (Favard's theorem) (cf. [3, 5, 9]) Let $\left\{\Phi_{n}\right\}_{n=0}^{\infty}$ be a PS. Then $\left\{\Phi_{n}\right\}_{n=0}^{\infty}$ is a WOPS relative to a quasi-definite moment functional $\sigma$ if and only if $D_{n}^{k}=0$ for $k=0,1, \cdots, n-2$ so that $\left\{\Phi_{n}\right\}_{n=0}^{\infty}$ satisfy a three term recurrence relation

$$
\mathbf{x} \Phi_{n}(\mathbf{x})=A_{n} \Phi_{n+1}(\mathbf{x})+B_{n} \Phi_{n}(\mathbf{x})+C_{n} \Phi_{n-1}(\mathbf{x}), n \geq 0\left(\Phi_{-1}(\mathbf{x}) \equiv 0\right)
$$

and

$$
\operatorname{rank} \widetilde{C}_{n}=n+1, n \geq 1 \text {, }
$$

where $\widetilde{C}_{n}:=\left[C_{n 1}, C_{n 2}\right]$ is an $(n+1) \times 2 n$ matrix.

If the equation (1.1) has a PS $\left\{\Phi_{n}\right\}_{n=0}^{\infty}$ as solutions, then it must be of the form

$$
\begin{aligned}
L[u]= & \left(a x^{2}+d_{1} x+e_{1} y+f_{1}\right) u_{x x}+\left(2 a x y+d_{2} x+e_{2} y+f_{2}\right) u_{x y} \\
& +\left(a y^{2}+d_{3} x+e_{3} y+f_{3}\right) u_{y y}+\left(g x+h_{1}\right) u_{x}+\left(g y+h_{2}\right) u_{y}=\lambda_{n} u
\end{aligned}
$$


where $\lambda_{n}:=\operatorname{an}(n-1)+g n([5])$.

We always assume that $|A|+|B|+|C| \not \equiv 0$ since otherwise the equation (1.5) cannot have any OPS as solutions(cf.[1]). Following Krall and Sheffer[5], we also assume that the equation (1.5) is admissible, that is, $\lambda_{m} \neq \lambda_{n}$ for $m \neq n$ (or equivalently $a n+g \neq 0, n \geq 0$ ) so that the equation (1.5) has a unique monic PS as solutions.

LeMma 1.5. ([1, Lemma 3.1) If the equation (1.5) has a PS $\left\{\Phi_{n}\right\}_{n=0}^{\infty}$ as solutions, then the canonical moment functional $\sigma$ of $\left\{\Phi_{n}\right\}_{n=0}^{\infty}$ satisfies

$$
L^{*}[\sigma]:=(A \sigma)_{x x}+2(B \sigma)_{x y}+(C \sigma)_{y y}-(D \sigma)_{x}-(E \sigma)_{y}=0 .
$$

If we set $S_{n}:=\left\langle\sigma, \mathbf{x}^{n}\right\rangle, n \geq 0\left(S_{-1}=S_{-2}=0\right)$ then we may rewrite (1.6) as

$$
\left\langle L^{*}[\sigma], \mathbf{x}^{n}\right\rangle=\left\langle\sigma, \lambda_{n} \mathbf{x}^{n}+B_{n} \mathbf{x}^{n-1}+C_{n} \mathbf{x}^{n-2}\right\rangle=\lambda_{n} S_{n}+B_{n} S_{n-1}+C_{n} S_{n-2}=0,
$$

where

$$
\begin{aligned}
B_{k} & =D_{k}^{1} D_{k-1}^{1}\left(d_{1} M_{k-2}^{1}+e_{1} M_{k-2}^{2}\right)+D_{k}^{1} D_{k-1}^{2}\left(d_{2} M_{k-2}^{1}+e_{2} M_{k-2}^{2}\right) \\
& +D_{k}^{2} D_{k-1}^{2}\left(d_{3} M_{k-2}^{1}+e_{3} M_{k-2}^{2}\right)+h_{1} D_{k}^{1}+h_{2} D_{k}^{2} \\
C_{k} & =f_{1} D_{k}^{1} D_{k-1}^{1}+f_{2} D_{k}^{1} D_{k-1}^{2}+f_{3} D_{k}^{2} D_{k-1}^{2}
\end{aligned}
$$

and $x \mathbf{x}^{n}=M_{n}^{1} \mathbf{x}^{n+1}, y \mathbf{x}^{n}=M_{n}^{2} \mathbf{x}^{n+1}, \partial_{x} \mathbf{x}^{n}=D_{n}^{1} \mathbf{x}^{n-1}, \partial_{y} \mathbf{x}^{n}=D_{n}^{2} \mathbf{x}^{n-1}$. Here, $I_{n}$ is the $n \times n$ identity matrix and

$$
\begin{array}{ll}
M_{n}^{1}=\left[I_{n+1} \mid 0\right], & M_{n}^{2}=\left[0 \mid I_{n+1}\right] \\
D_{n}^{1}=[\operatorname{Diag}(n, \cdots, 1) \mid 0]^{T}, & D_{n}^{2}=[0 \mid \operatorname{Diag}(1, \cdots, n)]^{T}
\end{array}
$$

The equation (1.7) is a three term recurrence relation for vector moments $\left\{S_{n}\right\}_{n=0}^{\infty}$ of $\sigma$.

Proposition 1.6. ([1, Theorem 3.7]) Let $\left\{\Phi_{n}\right\}_{n=0}^{\infty}$ be a PS satisfying an admissible equation (1.5) and $\sigma$ the canonical moment functional of $\left\{\Phi_{n}\right\}_{n=0}^{\infty}$. Then the following statements are all equivalent:

(i) $\left\{\Phi_{n}\right\}_{n=0}^{\infty}$ is a WOPS relative to $\sigma$;

(ii) $M_{1}[\sigma]:=(A \sigma)_{x}+(B \sigma)_{y}-D \sigma=0$;

(iii) $M_{2}[\sigma]:=(B \sigma)_{x}+(C \sigma)_{y}-E \sigma=0$.

Note that $L^{*}[\sigma]=\left(M_{1}[\sigma]\right)_{x}+\left(M_{2}[\sigma]\right)_{y}$. We call $M_{1}[\sigma]=0$ and $M_{2}[\sigma]=0$ the moment equations for the equation (1.5).

Using the moments $\sigma_{m n}$ of $\sigma$, we may express $L^{*}[\sigma]=0, M_{1}[\sigma]=0$, and $M_{2}[\sigma]=$ 0 as (cf. $[1,5])$

$$
\begin{aligned}
A_{m n}: & =\left\langle L^{*}[\sigma], x^{m} y^{n}\right\rangle=\frac{1}{2}\left(m C_{m-1, n}+n B_{m, n-1}\right) \\
B_{m n}: & =-2\left\langle M_{2}[\sigma], x^{m} y^{n}\right\rangle=2\{a(m+n)+g\} \sigma_{m, n+1}+e_{2} m \sigma_{m-1, n+1} \\
& +\left(d_{2} m+2 e_{3} n+2 h_{2}\right) \sigma_{m n}+f_{2} m \sigma_{m-1, n}+2 f_{3} n \sigma_{m, n-1}+2 d_{3} n \sigma_{m+1, n-1}=0 \\
C_{m n}: & =-2\left\langle M_{1}[\sigma], x^{m} y^{n}\right\rangle=2\{a(m+n)+g\} \sigma_{m+1, n}+\left(2 d_{1} m+e_{2} n+2 h_{1}\right) \sigma_{m n} \\
& +d_{2} n \sigma_{m+1, n-1}+2 f_{1} m \sigma_{m-1, n}+f_{2} n \sigma_{m, n-1}+2 e_{1} m \sigma_{m-1, n+1}=0, m \text { and } n \geq 0 .
\end{aligned}
$$




\section{Centrally symmetric OPS and Partial differential equations.}

Definition 2.1. We call a $P S\left\{\Phi_{n}\right\}_{n=0}^{\infty}$ to be centrally symmetric if $\Phi_{n}(-x,-y)$ $=(-1)^{n} \Phi_{n}(x, y), n \geq 0$. Also, we call a moment functional $\sigma$ to be centrally symmetric if $\left\langle\sigma, x^{m} y^{n}\right\rangle=0$ for $m+n$ odd.

LEMMA 2.2. ([10, Theorem 2.2.1]) Let $\left\{\Phi_{n}\right\}_{n=0}^{\infty}$ be a WOPS relative to a quasidefinite moment functional $\sigma$ so that (1.3) holds. Then the following statements are all equivalent:

(i) $\left\{\Phi_{n}\right\}_{n=0}^{\infty}$ is centrally symmetric;

(ii) $\sigma$ is centrally symmetric;

(iii) $B_{n}=0, n \geq 0$.

Furthermore, we have:

Proposition 2.3. Assume that the equation (1.5) has a WOPS $\left\{\Phi_{n}\right\}_{n=0}^{\infty}$ relative to a quasi-definite moment functional $\sigma$ as solutions. Then $\sigma$ is centrally symmetric if and only if the equation (1.5) is of the form

$$
L[u]=\left(a x^{2}+f_{1}\right) u_{x x}+\left(2 a x y+f_{2}\right) u_{x y}+\left(a y^{2}+f_{3}\right) u_{y y}+g\left(x u_{x}+y u_{y}\right)=\lambda_{n} u .
$$

Moreover, in this case, $\Delta:=f_{2}^{2}-4 f_{1} f_{3} \neq 0$.

Proof. It follows from (1.6), Proposition 1.3, and Proposition 1.6.

It's easy to see(cf. [5]) that under a real linear change of variables $T(x, y)=$ $(\alpha x+\beta y, \gamma x+\delta y), \alpha \delta-\beta \gamma \neq 0$, the equation (2.1) is transformed into

$$
L[u]=\left(a x^{2}+f_{1}^{*}\right) u_{x x}+\left(2 a x y+f_{2}^{*}\right) u_{x y}+\left(a y^{2}+f_{3}^{*}\right) u_{y y}+g\left(x u_{x}+y u_{y}\right)=\lambda_{n} u
$$

where $f_{1}^{*}=\alpha^{2} f_{1}+\alpha \beta f_{2}+\beta^{2} f_{3}, f_{2}^{*}=2 \alpha \gamma f_{1}+(\alpha \delta+\beta \gamma) f_{2}+2 \beta \delta f_{3}$, and $f_{3}^{*}=$ $\gamma^{2} f_{1}+\gamma \delta f_{2}+\delta^{2} f_{3}$.

Therefore, we may transform the equation (2.1) into either

$$
L[u]=a x^{2} u_{x x}+\left(2 a x y+f_{2}\right) u_{x y}+a y^{2} u_{y y}+g\left(x u_{x}+y u_{y}\right)=\lambda_{n} u
$$

if $\Delta>0$ or

$$
L[u]=\left(a x^{2}+f_{1}\right) u_{x x}+2 a x y u_{x y}+\left(a y^{2}+f_{1}\right) u_{y y}+g\left(x u_{x}+y u_{y}\right)=\lambda_{n} u,
$$

if $\Delta<0$ where $a=0$ or 1 and $f_{1} \neq 0, f_{2} \neq 0$ provided that $\Delta \neq 0$.

LEMMA 2.4. Assume that $\Delta:=f_{2}^{2}-4 f_{1} f_{3} \neq 0$. Then the (unique) monic $P S$ $\left\{\mathbb{P}_{n}\right\}_{n=0}^{\infty}$ of solutions to the equation (2.1) is always a WOPS.

Proof. Under the complex linear change of variables $T(x, y)=(x+i y, x-i y)$, the equation (2.4) is transformed into the equation (2.3). Since weak orthogonality is preserved under any linear change of variables, we may consider only the equation (2.3). Let $\sigma$ be the canonical moment functional of the monic PS $\left\{\mathbb{P}_{n}\right\}_{n=0}^{\infty}$ of solutions to the equation (2.3). Then by Lemma 1.5, $A_{m n}=0, m$ and $n \geq 0$ so that $\sigma_{n 0}=$ $\sigma_{0 n}=0, n \geq 1$. Hence $\sigma_{m n}=0$ for $m \neq n$ by induction. Then it's easy to see that $B_{m n}=0$, i.e., $M_{2}[\sigma]=0$ so that $\left\{\mathbb{P}_{n}\right\}_{n=0}^{\infty}$ is a WOPS relative to $\sigma$ by Proposition 1.6. 
THEOREM 2.5. The equation (1.5) has a centrally symmetric OPS as solutions if and only if the equation (1.5) is of the form (2.1) and $a \neq g, \Delta \neq 0$.

In order to prove Theorem 2.5, we need to extend Favard's theorem for WOPS's.

Proposition 2.6. Let $\left\{\Phi_{n}\right\}_{n=0}^{\infty}$ be a WOPS relative to $\sigma$. Then $\sigma$ is quasidefinite if and only if the rank condition (1.4) holds.

Proof. See the proof of Theorem 2 in [9](see also [4]).

Proof. [Proof of Theorem 2.5] Consider the equation (2.1) where $a \neq g$. We may assume that the equation (2.1) is of the form (2.3). Let $\left\{\mathbb{P}_{n}\right\}_{n=0}^{\infty}$ be the unique monic PS of solutions to the equation (2.3) and $\sigma$ the canonical moment functional of $\left\{\mathbb{P}_{n}\right\}_{n=0}^{\infty}$. Then by Lemma $2.4,\left\{\mathbb{P}_{n}\right\}_{n=0}^{\infty}$ is a WOPS relative to $\sigma$ so that it suffices to show the rank condition (1.4) holds for $\left\{\mathbb{P}_{n}\right\}_{n=0}^{\infty}$. We set $\mathbb{P}_{n}(\mathbf{x})=\sum_{k=0}^{n} A_{k}^{n} \mathbf{x}^{k}, n \geq 0$. Then we have $A_{n}^{n}=I_{n+1}, A_{n-1}^{n}=A_{n-3}^{n}=\cdots=0$ and $\left(\lambda_{n}-\lambda_{n-2}\right) A_{n-2}^{n}=f_{2} D_{n}^{1} D_{n-1}^{2}=$ $f_{2}[0|\operatorname{Diag}(n-1,2(n-2), \cdots,(n-2) 2, n-1)| 0]^{T}, n \geq 2$.

We also have

$$
\left\{\begin{array}{l}
x \mathbb{P}_{n}=A_{n 1} \mathbb{P}_{n+1}+C_{n 1} \mathbb{P}_{n-1}\left(\text { modulo } \mathcal{P}_{n-2}\right) \\
y \mathbb{P}_{n}=A_{n 2} \mathbb{P}_{n+1}+C_{n 2} \mathbb{P}_{n-1}\left(\text { modulo } \mathcal{P}_{n-2}\right)
\end{array}\right.
$$

where $A_{n j}=M_{n}^{j}, C_{n j}=A_{n-2}^{n} M_{n-2}^{j}-M_{n}^{j} A_{n-1}^{n+1}, n \geq 1\left(A_{-1}^{1}=0\right)$ and $j=1,2$. Hence

$$
\begin{aligned}
t_{n} t_{n+1} f_{2}^{-1} C_{n j} & =t_{n+1} D_{n}^{1} D_{n-1}^{2} M_{n-2}^{j}-t_{n} M_{n}^{j} D_{n+1}^{1} D_{n}^{2} \\
& = \begin{cases}{\left[0 \mid \operatorname{Diag}\left\{t_{n+1} k(n-k)-t_{n} k(n-k+1)\right\}_{k=1}^{n}\right]^{T}} & \text { for } j=1 \\
{\left[\operatorname{Diag}\left\{t_{n+1} k(n-k)-t_{n}(k+1)(n-k)\right\}_{k=1}^{n} \mid 0\right]^{T}} & \text { for } j=2,\end{cases}
\end{aligned}
$$

where $t_{n}=\lambda_{n}-\lambda_{n-2}, n \geq 1\left(\lambda_{-1}=0\right)$.

Note that

$$
\left|\operatorname{Diag}\left\{t_{n+1} k(n-k)-t_{n} k(n-k+1)\right\}_{k=1}^{n}\right|= \begin{cases}-\lambda_{1}=-g & \text { if } n=1 \\ \prod_{k=1}^{n} 2 k[(3-2 k) a-g] & \text { if } n \geq 2\end{cases}
$$

Hence, $\operatorname{rank} C_{n 1}=\operatorname{rank}\left(t_{n} t_{n+1} f_{2}^{-1} C_{n 1}\right)=n, n \geq 1$ since $a k+g \neq 0, k \geq-1$.

Similarly, $\operatorname{rank} C_{n 2}=n, n \geq 1$. In particular, $\operatorname{rank} \widetilde{C}_{n}=\operatorname{rank}\left(t_{n} t_{n+1} f_{2}^{-1} \widetilde{C}_{n}\right)=$ $n+1, n \geq 1$ since the first $n+1$ columns of $t_{n} t_{n+1} f_{2}^{-1} \widetilde{C}_{n}$ are linearly independent.

Conversely, assume that the equation (1.5) has a centrally symmetric OPS $\left\{\Phi_{n}\right\}_{n=0}^{\infty}$ as solutions. Then the equation (1.5) must be of the form (2.1) and $\Delta \neq 0$. Furthermore, we may assume that the equation (2.1) is of the form (2.3). Let $\left\{\mathbb{P}_{n}\right\}_{n=0}^{\infty}$ be the normalization of $\left\{\Phi_{n}\right\}_{n=0}^{\infty}$ and $\sigma$ the canonical moment functional of $\left\{\mathbb{P}_{n}\right\}_{n=0}^{\infty}$. Then $\left\{\mathbb{P}_{n}\right\}_{n=0}^{\infty}$ is a WOPS relative to $\sigma$, which is quasi-definite and we have (2.5) and (2.6). We now assume $a=g=1$. Then

$$
t_{2} t_{3} f_{2}^{-1} \widetilde{C}_{2}=-8\left[\begin{array}{cccc}
0 & 0 & 1 & 0 \\
0 & 0 & 0 & 0 \\
0 & 1 & 0 & 0
\end{array}\right]
$$

so that $\operatorname{rank} \widetilde{C}_{2}=2$, which contradicts Favard's theorem. 
By a suitable real linear change of variables, the differential equations (2.3) and (2.4) can be transformed into:

$$
\begin{aligned}
& L[u]=\left(x^{2}+1\right) u_{x x}+2 x y u_{x y}+\left(y^{2}-1\right) u_{y y}+g x u_{x}+g y u_{y} \\
& =n(g+n-1) u(\Delta>0, a \neq 0) \text {; } \\
& L[u]=u_{x x}-u_{y y}+2 x u_{x}+2 y u_{y}=2 n u(\Delta>0, a=0, g>0) ; \\
& L[u]=u_{x x}-u_{y y}-2 x u_{x}-2 y u_{y}=-2 n u(\Delta>0, a=0, g<0) ; \\
& L[u]=\left(x^{2}+1\right) u_{x x}+2 x y u_{x y}+\left(y^{2}+1\right) u_{y y}+g x u_{x}+g y u_{y} \\
& =n(g+n-1) u\left(\Delta<0, a \neq 0, a f_{1}>0\right) \text {; } \\
& L[u]=\left(x^{2}-1\right) u_{x x}+2 x y u_{x y}+\left(y^{2}-1\right) u_{y y}+g x u_{x}+g y u_{y} \\
& =n(g+n-1) u\left(\Delta<0, a \neq 0, a f_{1}<0\right) \text {; } \\
& L[u]=u_{x x}+u_{y y}+2 x u_{x}+2 y u_{y}=2 n u\left(\Delta<0, a=0, g f_{1}>0\right) ; \\
& L[u]=u_{x x}+u_{y y}-2 x u_{x}-2 y u_{y}=-2 n u\left(\Delta<0, a=0, g f_{1}<0\right) \text {. }
\end{aligned}
$$

By Theorem 2.5, above seven equations have centrally symmetric OPS's as solutions if and only if $g=1,0,-1, \cdots$.

Proposition 2.7. (cf. Proposition 4.1 and Theorem 4.5 in [1]) Let $\left\{\mathbb{P}_{n}\right\}_{n=0}^{\infty}$ be the monic PS of solutions to the equation (1.5) and $\sigma$ the canonical moment functional of $\left\{\mathbb{P}_{n}\right\}_{n=0}^{\infty}$. If $A_{y}=0$ (resp., $\left.C_{x}=0\right)$, then $P_{n 0}(x, y)=P_{n 0}(x)$ (resp., $P_{0 n}(x, y)=$ $\left.P_{0 n}(y)\right), n \geq 0$, and $\left\{P_{n 0}(x)\right\}_{n=0}^{\infty}$ (resp., $\left.\left\{P_{0 n}(y)\right\}_{n=0}^{\infty}\right)$ is a WOPS in one variable satisfying the equation

$$
A u_{x x}+D u_{x}=\lambda_{n} u\left(r e s p ., C u_{y y}+E u_{y}=\lambda_{n} u\right) .
$$

Moreover, if $\sigma$ is positive-definite, then $\left\{P_{n 0}(x)\right\}_{n=0}^{\infty}$ (resp., $\left.\left\{P_{0 n}(y)\right\}_{n=0}^{\infty}\right)$ is a positive-definite classical OPS in one variable. If $A_{y}=C_{x}=B=0$, then

$$
P_{m n}(x, y)=P_{m 0}(x) P_{0 n}(y), \quad m \text { and } n \geq 0 .
$$

Proposition 2.8. (cf. $[6,8]$ ) A second order ordinary equation

$$
\alpha(x) y^{\prime \prime}(x)+\beta(x) y^{\prime}(x)=\lambda_{n} y(x)
$$

where $\alpha(x)=a x^{2}+b x+c(\not \equiv 0), \beta(x)=d x+e$, and $\lambda_{n}=a n(n-1)+d n$, has an OPS (resp., a positive-definite OPS) as solutions if and only if for each $n \geq 0$

$$
s_{n}:=a n+d \neq 0 \text { and } \alpha\left(\frac{-t_{n}}{s_{2 n}}\right) \neq 0\left(\text { resp., } \frac{s_{n-1}}{s_{2 n-1} s_{2 n+1}} \alpha\left(\frac{-t_{n}}{s_{2 n}}\right)<0\right),
$$

where $t_{n}:=b n+e$.

By Propositions 2.7 and 2.8, the equations (2.7) (2.10) and (2.12) cannot have positive-definite OPS's as solutions. The equation (2.13) has a positive-definite OPS $\left\{H_{n-k}(x) H_{k}(y)\right\}_{k=0, n=0}^{n}$ as solutions, where $\left\{H_{n}(x)\right\}_{n=0}^{\infty}$ are Hermite polynomials. It is well known that the equation (2.11) has a positive-definite OPS, called the circle polynomials, as solutions for $g>1$. We now claim that the equation (2.11) has a positive-definite OPS as solutions only when $g>1$ (but has a quasi-definite OPS as solutions for $g \neq 1,0,-1, \cdots)$. Assume that the equation (2.11) has a positive-definite OPS as solutions. Then, by Propositions 2.7 and 2.8, $g>0$. We now let $\sigma$ be the 
canonical moment functional of the monic PS $\left\{\mathbb{P}_{n}\right\}_{n=0}^{\infty}$ of solutions to the equation (2.11). Then, we have from $A_{m n}=0$

$$
\begin{aligned}
& \sigma_{10}=\sigma_{01}=\sigma_{11}=\sigma_{30}=\sigma_{21}=\sigma_{12}=\sigma_{03}=\sigma_{31}=\sigma_{13}=0, \sigma_{20}=\sigma_{02}=1 /(g+1) \\
& \sigma_{40}=\sigma_{04}=3 \sigma_{22}=3 /(g+1)(g+3)
\end{aligned}
$$

so that $\Delta_{2}:=\operatorname{det} D_{2}=\frac{4(g-1)}{(g+1)^{6}(g+1)^{3}}$. Thus $g>1$ since $\Delta_{2}>0$.

In summary, we have proved:

COROLlary 2.9. The equation (2.1) has a positive-definite OPS as solutions if and only if either $\Delta<0, a f_{1}<0$, and ag $>1$ or $\Delta<0, a=0, g f_{1}<0$.

Allowing complex linear change of variables, Krall and Sheffer[5] found only the equations (2.11) and (2.13). We now give the explicit form of OPS $\left\{\Phi_{n}\right\}_{n=0}^{\infty}$ of solutions to each of the equations $(2.7) \sim(2.13)$ (see [2]).

- The equation (2.7):

$$
\phi_{n-k, k}(x, y)=\check{P}_{n-k}^{\left(\frac{g}{2}+k-1, \frac{g}{2}+k-1\right)}(x)\left(1+x^{2}\right)^{\frac{k}{2}} P_{k}^{\left(\frac{g}{2}-\frac{3}{2}, \frac{g}{2}-\frac{3}{2}\right)}\left(\frac{y}{\sqrt{1+x^{2}}}\right), 0 \leq k \leq n ;
$$

- The equation (2.8): $\quad \phi_{n-k, k}(x, y)=\check{H}_{n-k}(x) H_{k}(y), 0 \leq k \leq n$;

- The equation (2.9): $\quad \phi_{n-k, k}(x, y)=H_{n-k}(x) \check{H}_{k}(y), 0 \leq k \leq n$;

- The equation (2.10):

$$
\phi_{n-k, k}(x, y)=\check{P}_{n-k}^{\left(\frac{g}{2}+k-1, \frac{g}{2}+k-1\right)}(x)\left(1+x^{2}\right)^{\frac{k}{2}} \check{P}_{k}^{\left(\frac{g}{2}-\frac{3}{2}, \frac{g}{2}-\frac{3}{2}\right)}\left(\frac{y}{\sqrt{1+x^{2}}}\right), 0 \leq k \leq n ;
$$

- The equation (2.11):

$$
\phi_{n-k, k}(x, y)=P_{n-k}^{\left(\frac{g}{2}+k-1, \frac{g}{2}+k-1\right)}(x)\left(1-x^{2}\right)^{\frac{k}{2}} P_{k}^{\left(\frac{g}{2}-\frac{3}{2}, \frac{g}{2}-\frac{3}{2}\right)}\left(\frac{y}{\sqrt{1-x^{2}}}\right), 0 \leq k \leq n ;
$$

- The equation (2.12): $\quad \phi_{n-k, k}(x, y)=\check{H}_{n-k}(x) \check{H}_{k}(y), 0 \leq k \leq n$;

- The equation (2.13): $\quad \phi_{n-k, k}(x, y)=H_{n-k}(x) H_{k}(y), 0 \leq k \leq n$.

Here, $\left\{P_{n}^{(\alpha, \beta)}(x)\right\}_{n=0}^{\infty},\left\{\check{P}_{n}^{(\alpha, \beta)}(x)\right\}_{n=0}^{\infty}$, and $\left\{\check{H}_{n}(x)\right\}_{n=0}^{\infty}, n \geq 0$ are Jacobi, twisted Jacobi, and twisted Hermite polynomials(see [7]), respectively.

ACKNOWLEDGEMENTS. This work is partially supported by KOSEF(98-0701-0301-5) and Korea Ministry of Education(BK21-project). Authors thank the referees for their valuable comments.

\section{REFERENCES}

[1] Y. J. KIM, K. H. KWON, AND J. K. LeE, Orthogonal polynomials in two variables and second order partial differential equations, J. Comp. Appl. Math., 82 (1997), pp. 239-260.

[2] K. H. Kwon, J. K. LeE, AND L. L. LitTlejohn, Orthogonal polynomial eigenfunctions of second order partial differential equations, preprint.

[3] M. A. Kowalski, Algebraic characterization of orthogonality in the space of polynomials, in Lect. Notes Math. 1171, Springer-Verlag, Berlin, 1985, pp. 101-110.

[4] H. L. Krall and I. M. Sheffer, Differential equations of infinite order for orthogonal polynomials, J. Math. Anal. Appl., 74 (1966), pp. 135-172.

[5] H. L. Krall and I. M. Sheffer, Orthogonal polynomials in two variables, Ann. Mat. Pura Appl., seri 4, 76 (1967), pp. 325-376. 
[6] K. H. Kwon, J. K. LeE, AND B. H. Yoo, Characterizations of classical orthogonal polynomials, Results in Math., 24 (1993), pp. 119-128.

[7] K. H. KWON AND L. L. LitTLEJOHN, Classification of classical orthogonal polynomials, J. Korean Math. Soc., 34 (1997), pp. 973-1008.

[8] F. Marcellán, A. Branquinho, and J. Petronilho, Classical orthogonal polynomials: $A$ functional approach, Acta Appl. Math., 34 (1994), pp. 283-303.

[9] Y. XU, On multivariate orthogonal polynomials, SIAM J. Math. Anal., 24 (1993), pp. 783-794.

[10] Y. XU, Common Zeros of Polynomials in Several Variables and Higher Dimensional Quadrature, Pitman Research Notes in Math. 312, 1994. 